\title{
RESEARCH ON THE MULTI-OBJECTIVE OPTIMIZED SCHEDULING OF THE FLEXIBLE JOB-SHOP CONSIDERING MULTI-RESOURCE ALLOCATION
}

\author{
Zhong, Y.; Li, J. M. \& Zhu, S. Z." \\ School of Computer and Information Engineering, Xiamen University of Technology, Xiamen \\ 361024, China \\ E-Mail: yzhong@xmut.edu.cn, lijm@xmut.edu.cn, szzhu@xmut.edu.cn (" Corresponding author)
}

\begin{abstract}
Targeting at the problems existing in the multi-objective scheduling of traditional flexible job shop and the complexity of multi-resource allocation, this paper establishes an improved calculation model considering the optimization of such four targets as completion time, labour distribution, equipment compliance and production cost. The multi-objective integrated constraint optimization algorithm is designed and the Pareto solution set following different rules based on the NSGA- $\Pi$ algorithm is finally obtained. The research results show that the centralized selection of processing equipment and low efficiency of the job sequencing in the scheduling of traditional flexible job shop get improved. The personnel scheduling in the flexible working resources is highlighted, and multi-rule dynamic programming is introduced to get the optimal completion time and personnel allocation program. The optimal scheduling program can be quickly searched out using the NSGA-П algorithm, which effectively improves the search efficiency. The batch production within certain range can reduce the product processing time, but at the same time, it will increase the manufacturing costs. The use of smooth movement can reduce the overall processing time, but a too small movement volume will cause the increase in the number of movements. The exact match between the operators, numerical control equipment and the product processing procedures contributes to the feasibility of the preproduction operation plan.

(Received, processed and accepted by the Chinese Representative Office.)
\end{abstract}

Key Words: Flexible Job Shop, Scheduling, Multi-Objective Optimization, Improved NSGA-П Algorithm, Multi-Resource

\section{INTRODUCTION}

The production scheduling problem of the job shop is to make a reasonable and orderly distribution of a variety of resources in the job shop, so that the production workshop can use the least cost to achieve the best production efficiency. Based on the traditional production scheduling, the flexible job-shop scheduling (FJSP) breaks through the limitation of the uniqueness of machine, and makes the manufacturing procedure completed by a variety of machines through collaboration. The application of flexible scheduling to allocate resources contributes to an exponential increase in the search space. Thus, it is of great significance in reducing the search time of FJSP, and improving the accuracy and efficiency of the job-shop scheduling [1-7].

In the actual production scheduling problem of the flexible job shop, the multi-objective and multi-resource production scheduling has always been the problem which restrains its further development. At present, the multi-objective production scheduling can be divided into two steps: optimization of the multi-objective scheduling plan and decision-making. The solution algorithm for the multi-objective flexible job-shop scheduling is generally divided into two categories: traditional methods and intelligent methods. The traditional methods include the optimization method [8], the simulation method [9] and the heuristic rule scheduling method [10]; the intelligent algorithm mainly includes the intelligent calculation method and the intelligent search algorithm. The intelligent calculation method is divided into 
artificial neural network, evolutionary computation [11-14], swarm intelligence [15-17], artificial immune system [18], etc. The intelligent search algorithm mainly includes tabu search [19], simulated annealing [20], variable neighbourhood search [21] and so on.

Targeting at the problems existing in the multi-objective scheduling of traditional flexible job shop and the complexity of multi-resource allocation, this paper establishes an improved calculation model considering the optimization of such four targets as completion time, labour distribution, equipment compliance and production cost. The multi-objective integrated constraint optimization algorithm is designed and the Pareto solution set following different rules based on the NSGA- $\Pi$ algorithm is finally obtained. The effectiveness of the algorithm proposed in this paper is verified through examples.

\section{MULTI-OBJECTIVE OPTIMIZATION FOR FLEXIBLE JOB-SHOP SCHEDULING}

\subsection{Problem description and optimization model}

Let there be $N$ sets of equipment to produce the product $P$ and $L$ working procedures will be needed in total. Make the following assumptions during the production: (1) The processing route of the product is fixed; (2) The start time and stop time of the processing is confirmed; (3) The product can only be processed by a single machine at the same time; (4) The processing is set as non-preemptive, and the product has no priority; (5) The next working procedure shall not be started until the former one is completed.

Based on the above assumptions, after the processing sequence and the operation staff are confirmed, multiple scheduling objectives will be optimized simultaneously. Let the product set be $P=\{p=1,2,3, \ldots, P\}$, the working procedure set be $L=\{l=1,2,3, \ldots, L\}$, the equipment set be $M=\{m=1,2,3, \ldots, M\}$ and the operator set be $B=\{r=1,2,3, \ldots, B\}$.The multi-objective optimization model can be expressed as follows:

$$
\begin{gathered}
F_{1}=\min \left\{\sum_{p=1}^{P} \sum_{m=1}^{M} \sum_{l}^{L}\left[\max \left(s_{p m l}, t_{p m l}, f_{p m l}+\sum_{p=1}^{P} \sum_{m=1}^{M} \sum_{l}^{L} s_{p m l}\right)\right] x_{p m l}\right\} \\
F_{2}=\min \left\{\max \sum_{p=1}^{P} \sum_{m=1}^{M} \sum_{l}^{L} t_{p m l}\left(b_{l}\right) y_{b l}, f_{l+1}\left(B_{l+1}-b_{l}\right)\right\} \\
F_{3}=\min \left\{\max \sum_{p=1}^{P} \sum_{m=1}^{M} \sum_{l}^{L} x_{p m l} t_{p m l}\right\} \\
F_{4}=\min \left\{\max \sum_{p=1}^{P} \sum_{m=1}^{M} \sum_{l}^{L} x_{p m l} C_{t_{p m l}}\right\}
\end{gathered}
$$

Eqs. (1) to (4) respectively represent the minimum time needed to achieve the optimal production efficiency, the minimum number of staff needed for the minimum time, the maximum load of the equipment and the minimum production cost. $F_{1}-F_{4}$ are the objective functions to be optimized in this paper. $s_{p m l}, t_{p m l}$ and $f_{p m l}$ represent the start time, processing time and completion time of the product processing.

The product scheduling in the job shop is based on the refined scheduling technology. The operation process is as shown in Fig. 1. It can be seen from the figure that the product can be classified into three sub-batches $J_{1}-J_{3} p$ for processing. The same kind of equipment and operation staff will be arranged to conduct parallel operation. The total processing time can be divided into adjusting time and processing time, which can be separated. Meanwhile, the overall production time can be further shortened relying on the cross operation in the job shop 
through smooth movement. And the clearance squeezing method can reduce the idle time of the equipment and works, thus guaranteeing the production efficiency.

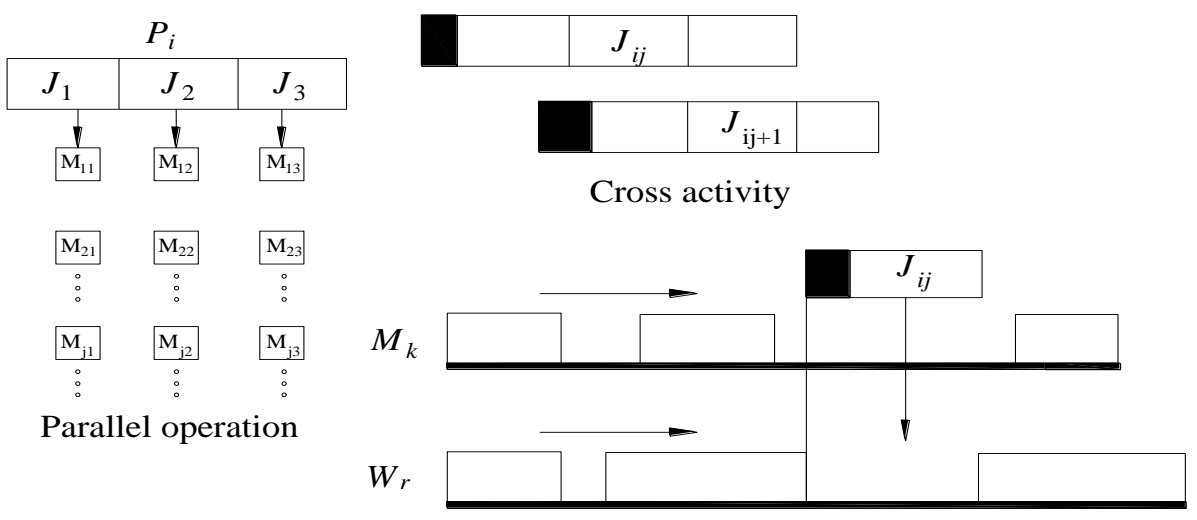

Clearance extrusion

Figure 1: The refinement scheduling.

The optimized selective probability can be expressed as:

$$
r r(i)\left(0<\operatorname{rr}(i) \leq 1, i=1,2, \ldots, c, \text { and } \sum_{i=1}^{c} r(i)=1\right)
$$

According to Eq. (5), the optimization objective which appears with great probability is more likely to be selected, i.e. assisting in the multi-objective optimization direction. The global optimal solution is obtained through following some kind of bulk selection rules. The management personnel can also allocate the resources and set the selective probability for the objectives to be optimized to find the satisfactory solution to the proposed algorithm as soon as possible.

Since the personnel assignment shall follow multiple rules, the dynamic programming algorithm is introduced to transform the multi-objective optimization problem to the continuous recursion problem. Let product $p$ be finished through $I$ working procedures in total, and take this process as one stage; the number of personnel $(B)$ is the status variable and the decision variable is the arrangement of a worker to procedure $I$ during the production. Then, the state transition equation and the phase indicator function can be respectively expressed as:

$$
\begin{gathered}
B_{l}=B_{(l+1) m}-b_{l} y_{l} \\
T_{l}\left(B_{l}\right)=\max \left\{T_{l}\left(b_{l} y_{b l}\right)\right\}
\end{gathered}
$$

On the basis of Eqs. (6) and (7), seek the solution to the optimal indicator function of the multiple objectives, i.e. the minimum completion time needed to complete the task under the multi-indicator decision scheme. There is:

$$
f_{l}\left(B_{l}\right)=\min \left\{\max \sum_{p=1}^{P} \sum_{m=1}^{M} \sum_{l}^{L} t_{p m l}\left(b_{l}\right) y_{b l}, f_{l+1}\left(B_{l+1}-b_{l}\right)\right\}
$$

\subsection{Multi-objective optimization algorithm following multiple rules}

Based on the above discussion, the improved NSGA- $\Pi$ flexible job-shop multi-objective scheduling optimization algorithm is designed. The main contents of the optimization are as follows: (1) coding the processing sequence of each product, which reduced the computational complexity; (2) allocation of resources based on multiple rules; (3) design of a new genetic operator, and the adoption of the exchange mutation operator in mutation 
operation. The scheduling problem in the flexible workshop is transformed into such key issues as chromosome coding, congestion degree calculation, target value seeking and decoding. The flow of the algorithm is shown in Fig. 2.

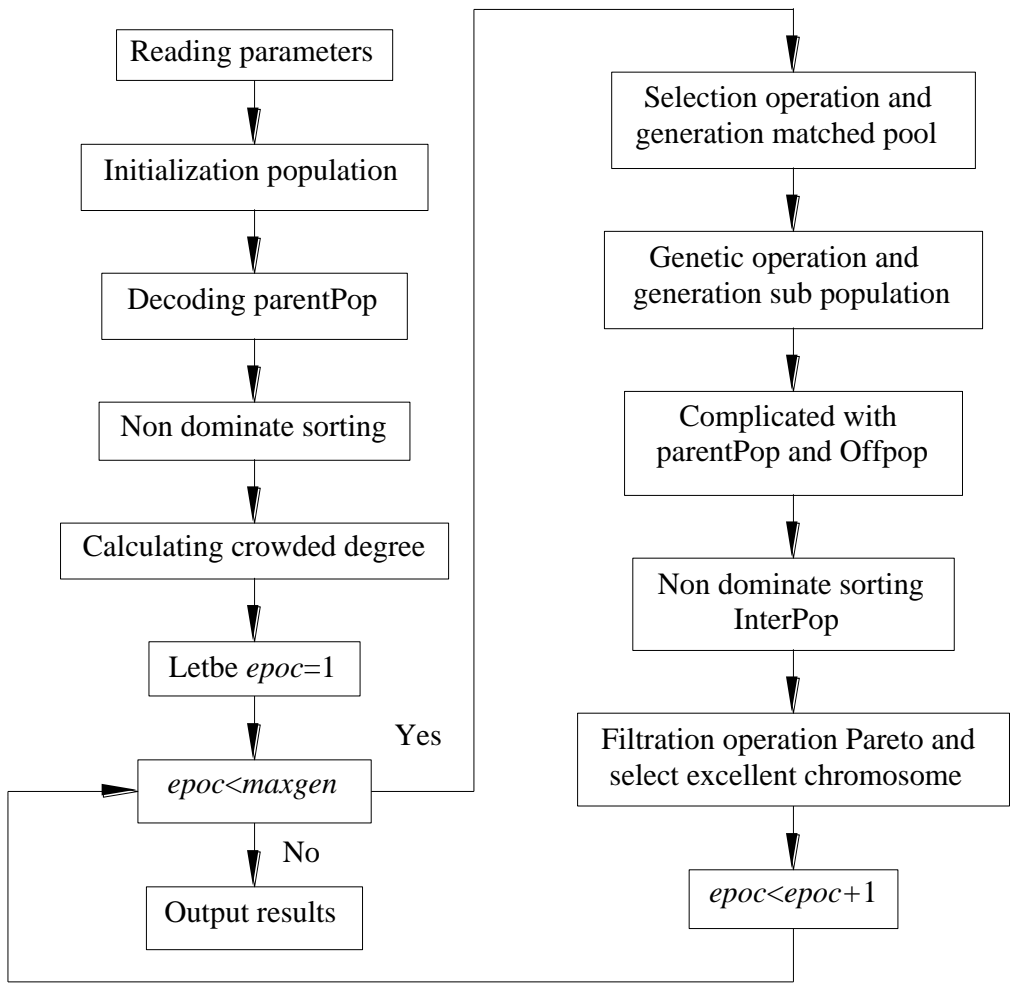

Figure 2: The flow chart of total optimization algorithm.

Table I: Job shop scheduling of $3 \times 4$ description.

\begin{tabular}{|c|c|c|c|c|c|}
\hline \multirow{2}{*}{ Product } & \multicolumn{5}{|c|}{ Equipment } \\
\cline { 3 - 6 } & & 1 & 2 & 3 & 4 \\
\hline \multirow{2}{*}{$P_{1}$} & $K_{11}$ & 4 & 2 & 4 & 3 \\
\cline { 2 - 6 } & $K_{12}$ & 3 & 5 & 5 & 2 \\
\hline \multirow{3}{*}{$P_{2}$} & $K_{21}$ & - & - & - & - \\
\cline { 2 - 6 } & $K_{22}$ & 5 & 4 & 5 & 6 \\
\cline { 2 - 6 } & $K_{23}$ & 3 & 5 & 5 & - \\
\hline \multirow{3}{*}{$P_{3}$} & $K_{31}$ & 5 & 5 & 6 & 7 \\
\cline { 2 - 6 } & $K_{32}$ & 6 & - & 4 & - \\
\cline { 2 - 6 } & $K_{33}$ & 4 & 4 & 5 & 5 \\
\hline
\end{tabular}

Table I shows a $3 \times 4$ scheduling example for a flexible job shop. The product processing adopts the process encoding way. The processing sequence number for each chromosome is composed by a number of natural numbers. If one of the processes of a product cannot be completed on the device $m$, the corresponding position is marked with "-".

In this paper, the improved NSGA- $\Pi$ flexible job-shop multi-objective scheduling optimization algorithm optimizes the concept of congestion and calculates the congestion degree of individuals using the method of interpolation.

$$
\operatorname{chr}(i) C_{d}=\sum_{k=1}^{M} \frac{f_{k}(i+1)-f_{k}(i-1)}{f_{k}^{\max }-f_{k}^{\min }}
$$

$f_{k}$ is a different objective function. $f_{1}, f_{2}, f_{3}$ and $f_{4}$ have the same meaning as those of Eqs. (1), (2), (3) and (4). $f_{k}(i+1)$ and $f_{k}(i-1)$ are the sum of the sides of the rectangular formed by 
the individual at the same level of adjacent points. The improved NSGA- $\Pi$ algorithm ensures that the algorithm finally converged to the uniformly distributed Pareto surface through the congestion degree. The larger the value of $\operatorname{chr}(i)$, the more sparse the surroundings of the point is, thus ensuring the diversity of the population.

\section{SIMULATION RESULTS VERIFICATION AND ANALYSIS}

\subsection{Simulation results comparison}

The proposed algorithm is programmed using Matlab, and applied in the actual scheduling of a job shop for verification and analysis. The parameters and the optimization model of the algorithm were debugged before the verification. Fig. 3 shows the comparison of the evolution curves of the iterations of and the minimum time needed for manufacturing 8 kinds of and 15 kinds of products using the algorithm proposed in this paper and the traditional jobshop scheduling algorithm. As can be seen from the figure, when the production equipment is fixed, the evolution curve is obtained using different scheduling and evolutionary algorithms. Compared with the traditional algorithm, the improved algorithm proposed in this paper can quickly converge in the $40^{\text {th }}$ generation when there are 8 kinds of products. When there are 15 kinds of products, it quickly converges in the $34^{\text {th }}$ generation. And the traditional algorithm converges in the $55^{\text {th }}$ generation and in the $50^{\text {th }}$ generation respectively when there are 8 kinds of products and 15 kinds of products. The distribution of products and equipment meets the constraints of the algorithm, and products can be collectively processed, and the occupancy of the equipment resource is fairly balanced.

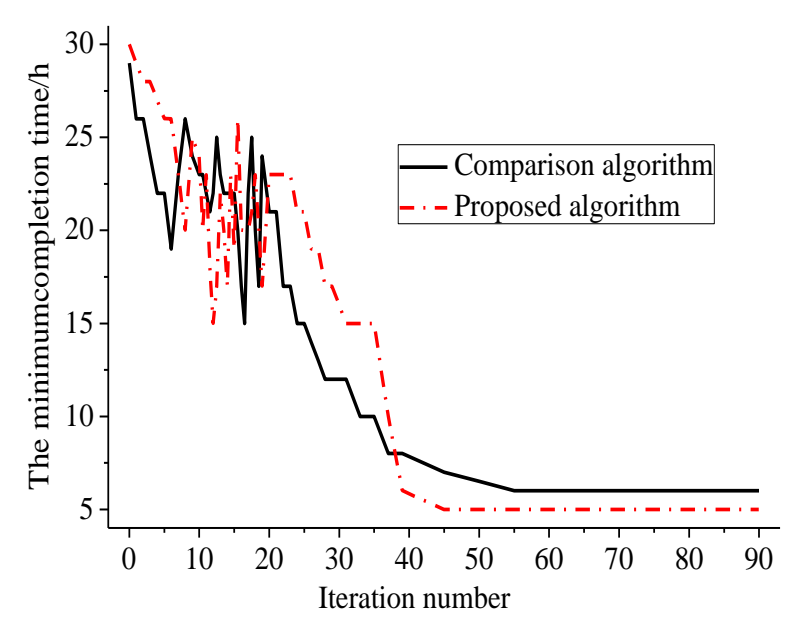

a) Simulation results of 8 products

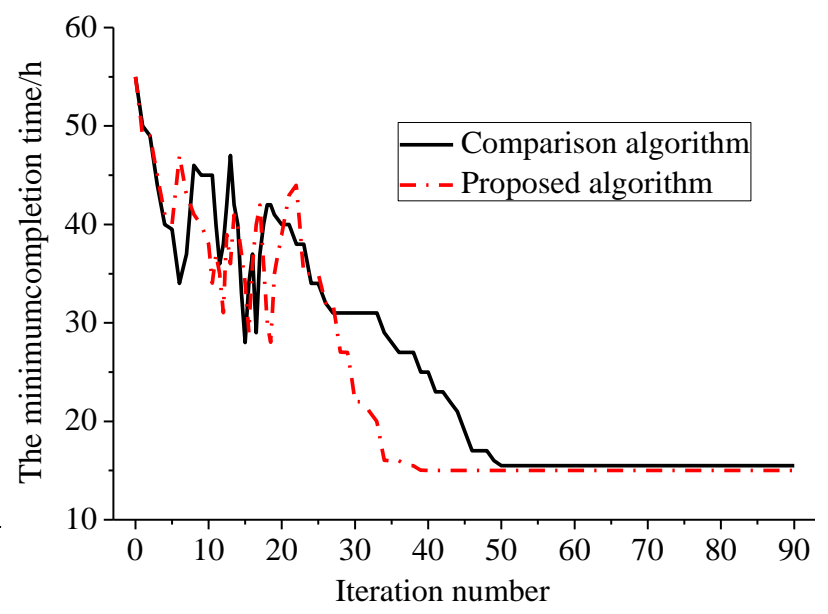

b) Simulation results of 15 products

Figure 3: Evolution curve of using different scheduling rules and algorithms to handle different product categories.

Table II shows the convergence algebra when the improved algorithm runs 15 times. It is seen from the table that the convergence algebra using the method proposed in this paper is much smaller than that using the traditional algorithm. Fig. 4 refers to the relationship between the number of the iterations and the minimum completion time calculated using the two algorithms. It can be seen from the figure that the algorithm proposed in this paper can meet the convergence requirement of the minimum completion time in the $16^{\text {th }}$ generation, while the traditional algorithm requires 34 generations to achieve the minimum completion time requirement. The traditional algorithm does not take into account the impact of personnel allocation on production efficiency, which is also the drawback of previous single resource analysis. 
Table II: $15^{\text {th }}$ simulation results.

\begin{tabular}{|c|c|c|c|c|c|}
\hline Serial number & 1 & 2 & 3 & 4 & 5 \\
\hline Convergence generation & 5 & 4 & 10 & 6 & 1 \\
\hline Serial number & 6 & 7 & 8 & 9 & 10 \\
\hline Convergence generation & 5 & 3 & 2 & 3 & 6 \\
\hline Serial number & 11 & 12 & 13 & 14 & 15 \\
\hline Convergence generation & 4 & 6 & 1 & 9 & 4 \\
\hline
\end{tabular}

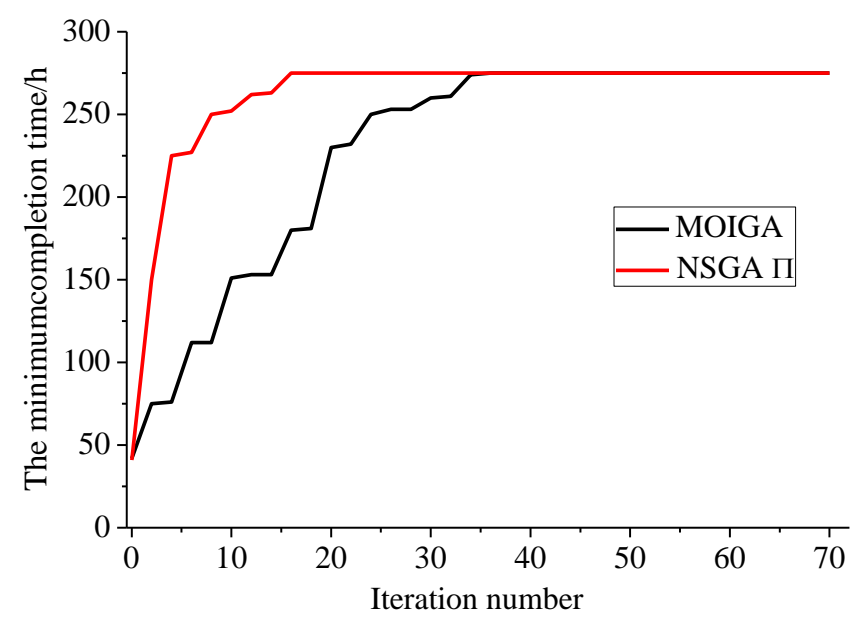

Figure 4: Impact of minimum completion time on different algorithm.

\subsection{Application verification}

In the practical application verification, a flexible mechanical job shop is selected as the engineering background. A total of 10 numerically-controlled machine tools are needed for the production, with the serial number of D001-D010. There are seven equipment operators, whose serial number is from W001 to W007. There are four kinds of products in total, and relevant processing parameters for each product are shown in Table III, where $C_{t}$ represents for the total time required for processing each product and $S_{t}$ stands for the adjustment time for processing products. The unit of $C_{t}$ and $S_{t}$ are both h. $P_{c}$ and $P_{s}$ mean the processing costs and adjustment costs in unit time. It can be seen from the table that the total time of product processing is separated into the sum of $C_{t}$ and $S_{t}$, and the difference of operators in the proficiency in operating the $\mathrm{NC}$ equipment makes the adjustment period of the product processing time different, thus refined matching is implemented among the equipment, the operator and the production process.

Table III: Processing technology parameters.

\begin{tabular}{|c|c|c|c|c|c|c|c|c|c|c|c|c|c|}
\hline \multirow{2}{*}{ Workers } & \multicolumn{4}{|c|}{ D001 } & \multicolumn{4}{|c|}{ D002 } & $\ldots$ & \multicolumn{4}{|c|}{ D010 } \\
\hline & $C_{t}$ & $S_{t}$ & $P_{c}$ & $P_{s}$ & $C_{t}$ & $S_{t}$ & $P_{c}$ & $P_{s}$ & $\ldots$ & $C_{t}$ & $S_{t}$ & $P_{c}$ & $P_{s}$ \\
\hline W001 & 0.51 & 2.1 & 81 & 78 & & & & & $\ldots$ & & & & \\
\hline W002 & 0.5 & 2.3 & 79 & 75 & & & & & $\ldots$ & & & & \\
\hline W003 & & & & & 0.51 & 2.7 & 75 & 71 & $\ldots$ & & & & \\
\hline W004 & & & & & 0.52 & 3.2 & 71 & 71 & $\ldots$ & 0.7 & 4.6 & 49 & 45 \\
\hline W005 & 0.5 & 3.1 & 73 & 70 & 0.53 & 3.5 & 70 & 69 & $\ldots$ & 0.68 & 5.1 & 46 & 43 \\
\hline W006 & 0.51 & 3.5 & 72 & 67 & & & & & $\ldots$ & 0.7 & 5.2 & 43 & 41 \\
\hline W007 & 0.5 & 3.7 & 69 & 65 & & & & & $\ldots$ & 0.7 & 5.7 & 42 & 41 \\
\hline
\end{tabular}

Four typical isometric batch schemes are established to verify the effect of different batches on the production efficiency. The batch size in the four schemes is 120-100-80-100, $60-50-40-50,30-25-20-25$ and 20-20-20-20 respectively. The moving batch and the 
processing batch are the same, and the completion time and the manufacturing cost curve under the four processing batches are shown in Fig. 5. It can be seen from the figure that within certain limits, the batch size is proportional to the completion time, i.e. the greater the batch size is, the longer the product completion time is. At the same time, the batch size is inversely proportional to the manufacturing cost. The reason is that with the decrease of the processed goods, the sub-batch size increases, so that the processing of the same product may be divided into multiple batches for parallel operations, thereby shortening the product's completion time. When the batch size is too small, the reduced processing time due to parallel operation is not enough to make up for the extra time due to the adjustment of the production processes and ultimately extends the processing time. From the figure, we can see that 60-5040-50 is a reasonable product batch scheme.

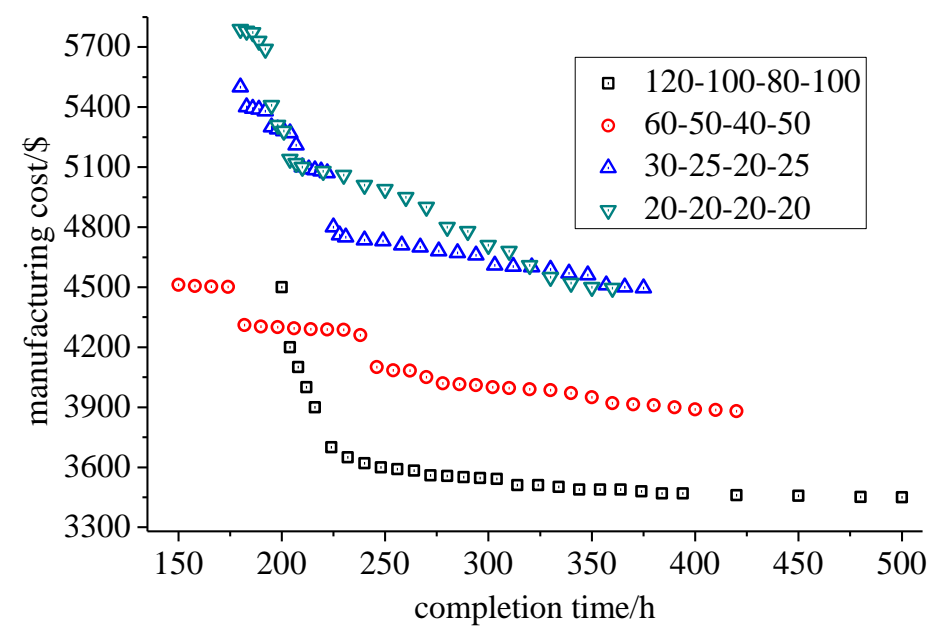

Figure 5: Variation of manufacturing cost with different manufacturing batch size.

Three kinds of movement batch schemes are established to verify the effect of the smooth movement of the product processing on the production efficiency. The size of each batch is 60-50-40-50, 15-10-10-10 and 5-5-5-5. The completion time of the three kinds of movement batch schemes and the production cost are shown in Fig. 6. It can be seen from the figure that the transformation of the processing batch schemes into the movement batch schemes through smooth movement can effectively reduce the time needed for the scheduling. The size of the movement batch shall not be too small; otherwise it will lead to the increase in the frequency of movement, which will further increase the workload of the handling personnel, while the completion time does not decrease obviously.

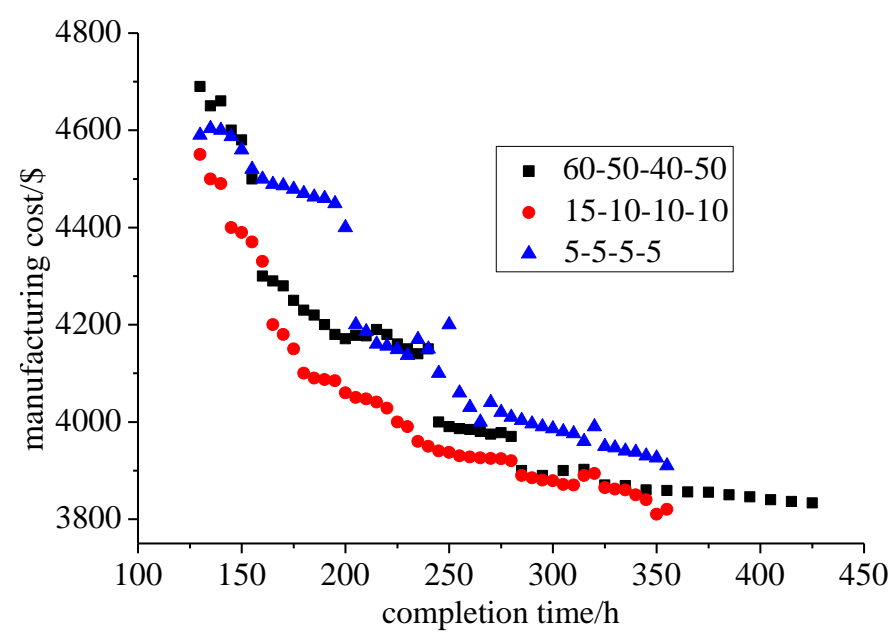

Figure 6: Variation of manufacturing cost with different movement batch size. 
Fig. 7 shows the technician sub-batch Gantt chart corresponding to certain Pareto solution. Each small area in the figure represents the production planning and scheduling of a subbranch. $r$ represents the production process. 1.1.1 stands for the first working procedure in the first processing batch of the product with the code of 1, and so on for other numbers. D003 means that the second process of the product 1 is arranged on equipment 3 . W005 means that the process is completed by the fifth operator. 46.6-85.6 refers to that the time required to finish this process ranges from 46.6 hours to 85.6 hours. It can be seen from the figure that there are overlaps between partial procedures, which is due to the smooth movement of the algorithm at the time of decoding, so that the next process of the same processing batch can be performed in advance, thus reducing the overall operating time. This once again proves the superiority of the improved algorithm proposed in this paper.

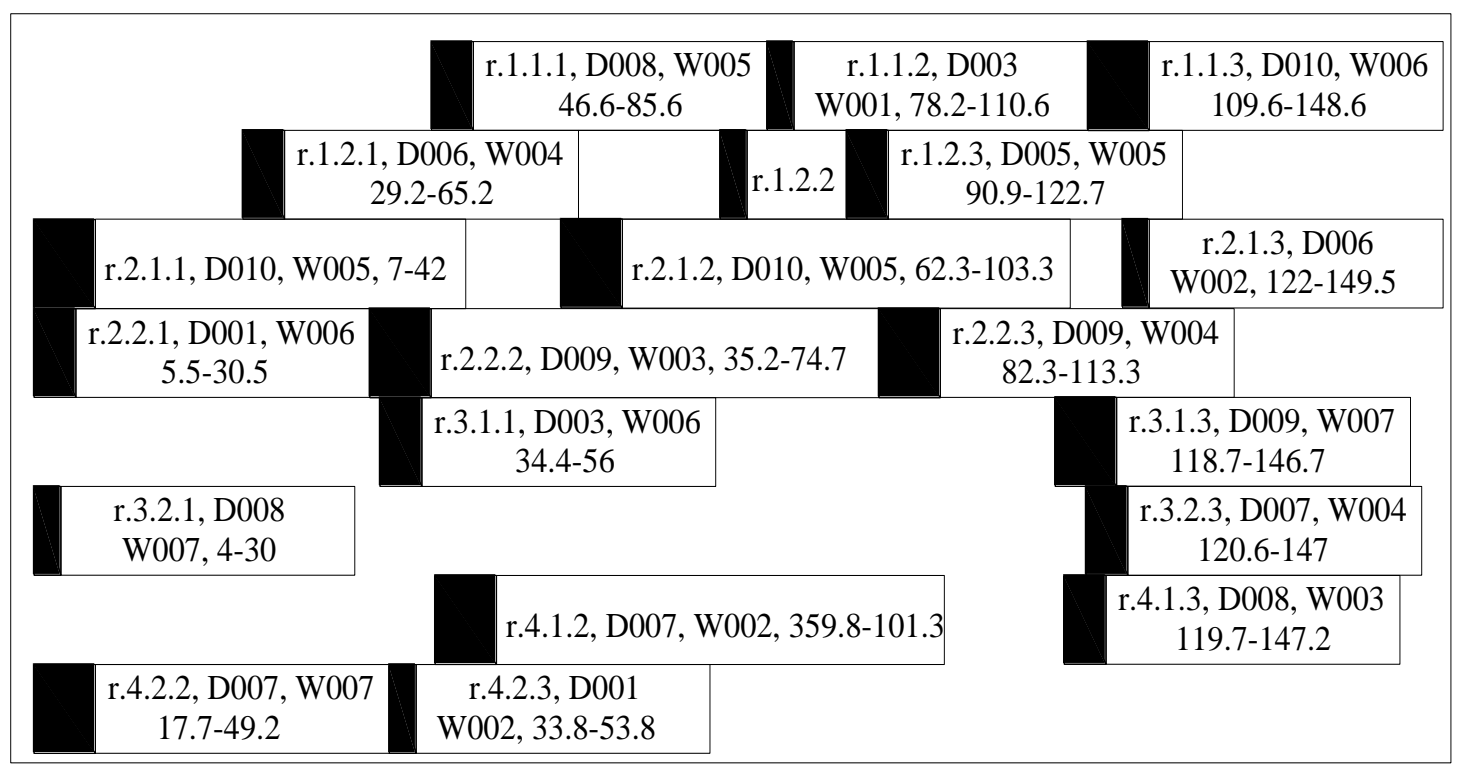

Figure 7: Processing sub-batch Gantt chart of a Pareto solution.

\section{CONCLUSIONS}

Targeting at the problems existing in the multi-objective scheduling of traditional flexible job shop and the complexity of multi-resource allocation, this paper establishes an improved calculation model considering the optimization of such four targets as completion time, labour distribution, equipment compliance and production cost. The multi-objective integrated constraint optimization algorithm is designed and the Pareto solution set following different rules based on the NSGA- $\Pi$ algorithm is finally obtained. The following conclusions are drawn:

(1) The centralized selection of processing equipment and low efficiency of the job sequencing in the scheduling of traditional flexible job shop get improved. The personnel scheduling in the flexible working resources is highlighted, and multi-rule dynamic programming is introduced to get the optimal completion time and personnel allocation program. The optimal scheduling program can be quickly searched out using the NSGA-П algorithm, which effectively improves the search efficiency.

(2) The batch production within certain range can reduce the product processing time, but at the same time, it will increase the manufacturing costs. The use of smooth movement can reduce the overall processing time, but a too small movement volume will cause the increase in the number of movements. The exact match between the operators, numerical control equipment and the product processing procedures contributes to the feasibility of the preproduction operation plan. 


\section{ACKNOWLEDGEMENTS}

Supported by Natural Science Foundation of P. R. China (No. 61373147, 61503316), Fujian Province Science and Technology Plan Project (No. 2016Y0079, 2016I0015), Xiamen Science and Technology Plan of University Innovation Project (No. 3502Z20153020).

\section{REFERENCES}

[1] Chen, J. C.; Wu, C. C.; Chen, C. W.; Chen, K. H. (2012). Flexible job shop scheduling with parallel machines using genetic algorithm and grouping genetic algorithm, Expert Systems with Applications, Vol. 39, No. 11, 10016-10021, doi:10.1016/j.eswa.2012.01.211

[2] Browning, T. R.; Yassine, A. A. (2010). Resource-constrained multi-project scheduling: priority rule performance revisited, International Journal of Production Economics, Vol. 126, No. 2, 212 228, doi:10.1016/j.ijpe.2010.03.009

[3] Yuan, Y.; Xu, H. (2013). Flexible job shop scheduling using hybrid differential evolution algorithms, Computers \& Industrial Engineering, Vol. 65, No. 2, 246-260, doi:10.1016/j.cie. 2013.02.022

[4] Chen, B.; Matis, T. I. (2013). A flexible dispatching rule for minimizing tardiness in job shop scheduling, International Journal of Production Economics, Vol. 141, No. 1, 360-365, doi:10.1016/j.ijpe.2012.08.019

[5] Tasan, S. O.; Gen, M. (2013). An integrated selection and scheduling for disjunctive network problems, Computers \& Industrial Engineering, Vol. 65, No. 1, 65-76, doi:10.1016/j.cie. 2011.12.022

[6] Lerher, T.; Ekren, Y. B.; Sari, Z.; Rosi, B. (2015). Simulation analysis of shuttle based storage and retrieval systems, International Journal of Simulation Modelling, Vol. 14, No. 1, 48-59, doi:10.2507/ijsimm14(1)5.281

[7] Wu, W.; Li, P. K.; Zhang, Y. (2015). Modelling and simulation of vehicle speed guidance in connected vehicle environment, International Journal of Simulation Modelling, Vol. 14, No. 1, 145-157, doi:10.2507/ijsimm14(1)co3

[8] Liao, S. H.; Hsieh, C. L.; Lai, P. J. (2011). An evolutionary approach for multi-objective optimization of the integrated location-inventory distribution network problem in vendormanaged inventory, Expert Systems with Applications, Vol. 38, No. 6, 6768-6776, doi: $10.1016 /$ j.eswa.2010.12.072

[9] Konak, A.; Coit, D. W.; Smith, A. E. (2006). Multi-objective optimization using genetic algorithms: a tutorial, Reliability Engineering \& System Safety, Vol. 91, No. 9, 992-1007, doi:10.1016/j.ress.2005.11.018

[10] Xiong, J.; Xing, L. N.; Chen, Y. W. (2013). Robust scheduling for multi-objective flexible jobshop problems with random machine breakdowns, International Journal of Production Economics, Vol. 141, No. 1, 112-126, doi:10.1016/j.ijpe.2012.04.015

[11] Xing, L. N.; Chen, Y. W.; Yang, K. W. (2009). Multi-objective flexible job shop schedule: design and evaluation by simulation modeling, Applied Soft Computing, Vol. 9, No. 1, 362-376, doi:10.1016/j.asoc.2008.04.013

[12] Li, J. Q.; Pan, Q. K.; Xie, S. X. (2010). A hybrid variable neighborhood search algorithm for solving multi-objective flexible job shop problems, Computer Science and Information Systems, Vol. 7, No. 4, 907-930, doi: 10.2298/csis0906080171

[13] Gao, J.; Sun, L.; Gen, M. (2008). A hybrid genetic and variable neighborhood descent algorithm for flexible job shop scheduling problems, Computers \& Operations Research, Vol. 35, No. 9, 2892-2907, doi:10.1016/j.cor.2007.01.001

[14] Wang, X.; Li, W.; Zhang, Y. (2013). An improved multi-objective genetic algorithm for fuzzy flexible job-shop scheduling problem, International Journal of Computer Applications in Technology, Vol. 47, No. 2/3, 280-288, doi:10.1504/ijcat.2013.054360

[15] Frutos, M.; Olivera, A. C.; Tohme, F. (2010). A memetic algorithm based on a NSGAII scheme for the flexible job-shop scheduling problem, Annals of Operations Research, Vol. 181, No. 1, 745-765, doi:10.1007/s10479-010-0751-9 
[16] Zeng, Q.; Yang, Y.; Cheng, B.; Yang, J. (2012). Research on multi-objective optimization for equal batch splitting FJSP under parallel and sequence shift mode, Journal of System Simulation, Vol. 24, No. 5, 1046-1052

[17] Grobler, J.; Engelbrecht, A. P.; Kok, S.; Yadavalli, S. (2010). Metaheuristics for the multiobjective FJSP with sequence-dependent set-up times, auxiliary resources and machine down time, Annals of Operations Research, Vol. 180, No. 1, 165-196, doi:10.1007/s10479-008-0501-4

[18] Liu, H.; Abraham, A.; Wang, Z. (2009). A multi-swarm approach to multi-objective flexible jobshop scheduling problems, Fundamenta Informaticae, Vol. 95, No. 4, 465-489, doi:10.3233/FI$\underline{2009-160}$

[19] Sun, W.; Pan, Y.; Lu, X.; Ma, Q. (2010). Research on flexible job-shop scheduling problem based on a modified genetic algorithm, Journal of Mechanical Science and Technology, Vol. 24, No. 10, 2119-2125, doi:10.1007/s12206-010-0526-x

[20] Tripathi, P. K.; Bandyopadhyay, S.; Pal, S. K. (2007). Multi-objective particle swarm optimization with time variant inertia and acceleration coefficients, Information Sciences, Vol. 177, No. 22, 5033-5049, doi:10.1016/j.ins.2007.06.018

[21] Xia, W.; Wu, Z. (2005). An effective hybrid optimization approach for multi-objective flexible job-shop scheduling problems, Computers \& Industrial Engineering, Vol. 48, No. 2, 409-425, doi: $\underline{10.1016 / j . c i e .2005 .01 .018}$ 